American Medical Journal 2 (2): 72-78, 2011

ISSN 1949-0070

(C) 2011 Science Publications

\title{
The Impact of Health Technology Assessment on Decision-Making Processes in Public Versus Not-for-Profit Private Hospitals
}

\author{
Hafizah Besar Saaid, Donald Stewart, Ian England and Nilesh Parmar \\ School of Public Health, Health Griffith, Griffith University-Logan Campus, \\ University Dr, Meadowbrook 4131 QLD, Australia
}

\begin{abstract}
Problem statement: The goal of this study is to extend research on Evidence-Based Practice (EBP) implementation by examining the decision-making processes for acquiring new health technologies in selected hospitals in Southeast Queensland, Australia. Both a decision-making model and a mini-Health Technology Assessment (HTA) model guide the approach and analysis in this study. We anticipated that both public and private sector organisations would use HTA as the guideline in decision-making processes to acquire new health technologies. Approach: The data were collected using two methods; document analysis and in-depth, face-to-face interviews. The steps in decisionmaking processes to acquire new health technologies were identified through content and thematic analysis. The HTA process and mini-HTA checklist were used as a bench mark for decision-making processes. Results: Decision making processes were described as informal in not-for-profit private hospitals and as formal in public hospitals. Decisions in not-for-profit private hospitals were driven by business strategy and the cost effectiveness of the technologies. In the public hospital, however, the main factors were safety and clinical effectiveness although budget also has some impact. Decision makers in both types of hospitals were unclear about HTA and its agencies. They also were not aware of mini-HTA, even though they were searching for a suitable support tool for decision making. Conclusion: This study identified the impact of HTA and mini-HTA in public and private hospital settings. Findings from this study show that the evidence from HTA is not fully utilised by decision makers in the hospitals to make informed decisions. Health authorities should play a more active role in educating decision makers at hospital level regarding health technology assessment. Mini-HTA can be a beneficial tool for decision making processes at hospital level.
\end{abstract}

Key words: Health technology assessment, mini-HTA, decision making process, not-for-profit private hospital, public hospital, decision support tool

\section{INTRODUCTION}

Health technology undoubtedly produces many benefits in patient care and quality of life. However, there are concerns about the adoption of untested technologies, increasing health care expenditure and an unavoidable growth in end-user expectations. These concerns have led to increasing interest in Health Technology Assessment (HTA) over the last 20 years (Stevens et al., 2003). HTA is broadly based in terms of both its methods and applications. It can contain assessments of cost, effectiveness, safety, efficacy, and cost-effectiveness, as well as covering organisational, social, ethical and legal implications. It can be applied to equipment, drugs and procedures, as well as the organisational and support systems. It can include existing as well as new or emerging technologies (Haas et al., 2008).
The main purpose of HTA is to offer policy makers and funders, health professionals and health consumers essential information to recognize the advantages and comparative value of health technologies and to make informed decisions either on policy, funding or clinical issues (Australian Government, 2009).

However, concerns have been expressed about the capability of HTA agencies to enhance the use of their findings by decision makers and target audiences (Lehoux et al., 2005). This issue led HTA agencies to conduct experiments with different knowledge transfer strategies and evaluate their varying effectiveness (Hivon et al., 2005).

The concern now is that knowledge generated from HTA is still not incorporated as it should be into political, organizational and clinical decisions (Gagnon et al., 2006). Extensive studies have been conducted to

Corresponding Author: Hafizah Besar Saaid, School of Public Health, Health Griffith, Griffith University-Logan Campus, University Dr, Meadowbrook 4131 QLD, Australia 
find out the most effective dissemination strategies that should be used by HTA producers (Haines et al., 2004). However, there are remarkably few studies on the effectiveness of dissemination strategies from the users' viewpoint (Hivon et al., 2005).

Hospitals as providers of healthcare are the main buyers of equipment and they provide the bulk of costly, high-technology services, thus they are most important users. Also, 'HTA can be used by hospitals for guiding difficult choices, especially in balancing organizational and community needs' (Banta and Oortwijn, 2000).

The problem of unlimited demands in a situation of limited resources must lead to prioritization in health care systems. This problem has become acute in today's world of rapidly emerging advanced technology and extensive investigation is constantly being undertaken to find solutions to problems relating to health service priorities (Leggat et al., 2006). The need to set priorities is directly related to the problem of decision making (Gallego et al., 2008). The fundamental feature of decision making lies in the formulation of alternatives to meet the situation under consideration and in choosing between these alternatives, after evaluating their effectiveness in achieving the decision maker's goals and objectives (HBSP, 2006).

The decision making environment in health care systems has been described as different and complex (Gallego, 2006). Decisions in health care can involve clinical decisions such as patients choosing treatments, or they can also involve health care policy makers such as those relating to the Pharmaceutical Schedule (PHARMAC) (Gallego, 2006).

The problem of priority setting is still under ongoing debate and there is little consensus on the best method to carry it out (Kapiriri and Norheim, 2004). Also there is no consensus to date on what are the optimal criteria for setting priorities related to choosing new health technology. Noorani et al. (2007) have identified 59 unique priority-setting criteria in their review of eleven HTA agencies. These sets of criteria are undoubtedly useful for the decision makers when evaluating new health technology for adoption (Johnson-Masotti and Eva, 2005). However, to get the information about whether the health technology under consideration complies with the listed criteria, decision makers must turn to HTA. Sanders argues.

The key issues governing decisions regarding the appropriate adoption of health technologies are the cost-effectiveness and efficacy of the technologies; this is where decision and policy makers turn to Health Technology Assessment (HTA)' (Sanders, 2002).
Yet, studies show that the HTA findings are still not optimally disseminated to decision makers especially at institutional and individual levels (McGregor, 2006; Sorenson et al., 2008). McGregor suggested that 'Possible explanations for this finding are that the HTA process lacks the necessary mechanisms to translate evidence into policy and that there is inadequate contact of HTA producers with the decision makers who use them'.

Hospital-based HTA or "mini-HTA" has been recognised as one strategy to incorporate HTA into the decision making process at hospital level. Mini-HTA is a management and decision support tool based on the reasoning involved in HTAs. The concept of mini-HTA was introduced by the Copenhagen University Hospital and in 2000, the Danish Centre for Evaluation and Health Technology Assessment (DACEHTA) prepared a form and a guide to the acquisition of medical devices for hospitals. Since then mini-HTA has developed as a standardised form, or check list, with a number of queries relating to the prerequisites for and implications of using (new) health technology (Vestergaard et al., 2005). Mini-HTA can be used both for applications for equipment and for the introduction of new treatments (Ehlers et al., 2006). It can be conducted in a short time frame and gives an input for decisions at the time when needed (Vestergaard et al., 2005).

Most previous studies related to new health technologies focus only on the decision making environment in public health institutions (Mullen, 2004). With the increase in demand from consumers, public hospitals are not the only providers; the emergence of private health institutions has become inevitable. Private hospitals consist of single hospital operators, not-for-profit organisations, private health insurance funds and large listed public companies. In Australia, not-for-profit private hospitals are owned by religious, charitable, or community institutions (Perrott and Hughes, 2005). This study investigated decision making processes for introducing new health technologies in both public and not-for-profit private hospitals.

This research addresses three main questions:

- How is a decision on acquiring new health technologies made?

- Do decision makers use HTA as a decision support tool?

- Are there any differences between public and notfor-profit private hospitals in using HTA in their practices?

\section{MATERIALS AND METHODS}

Two studies-one using document analysis and the other using semi-structured in-depth face-to-face 
interviews-were conducted between May to December 2010 .

Research setting: The study took place at hospitals in South East Queensland, Australia. A multiple case study method has been applied rather than random sampling, due to the difficulty of getting agreement to participate from all hospitals in South East Queensland within the research timescale. Four hospitals were selected using a convenience sampling method. Three were not-for-profit private hospitals and one was a public hospital.

Participants and recruitment: Documents related to decision-making processes for introducing new health technologies were collected from all hospitals. The documents collected included product review forms, and examples of business cases. Minutes of meetings, although potentially relevant in the decision making process for acquiring new health technologies, were excluded as they were confidential.

Interviews were conducted with decision makers who were directly involved in the decision making process for acquiring new health technologies in these four hospitals. Twenty-one interviews were conducted; ten with administrative managers, six with nurse managers and five with medical managers. The participants were chosen by using an organisational structure framework and a list of key decision making roles provided by the General Managers of the hospitals.

Data collection and analysis: The documents were analysed using a comparative method, comparing the documents collected from hospitals with a mini-HTA checklist produced by the Danish Centre (DACEHTA). The mini-HTA checklist from DACEHTA was used as a benchmark because of its worldwide recognition as a decision making support tool. Similarities and differences between items in the product review form and the business case with the mini-HTA checklist were noted.

The interview guide was developed to ensure the capture of pertinent information and structural similarity of each interview. The guide consisted of four parts; decision making processes, evaluation processes, perception of HTA, and implementation and future improvement. The participants signed a consent form before the interview. The interviews were recorded with permission from the participants. The recorded interviews were transcribed by the interviewer. Transcripts were read through, and notes were made. Segments or paragraphs were coded and labelled. Coded segments were then compared to find similarities of ideas and events. The processes were repeated until all comments were assigned into categories (Silverman 2004).

Ethics: This study was approved by Griffith University Human Research Ethics Committee and was further endorsed by Queensland Health Metro South Human Ethics Committee. All participants signed written consents before participating. The interviews were all de-identified and the confidentiality of all data was ensured through password protected procedures.

\section{RESULTS}

The results presented here are from the two data sources; documents and interviews.

Hospital demographics: Four hospitals participated in this study. Three are not-for-profit private hospitals and one is a State funded public hospital. All hospitals serve the population of South East Queensland. Because of confidentiality, the hospitals are coded as Private A, Private $\mathrm{B}$, and Private $\mathrm{C}$ for the not-for-profit private hospitals and Public for the public hospital. The sizes of hospitals are provided in Table 1.

Health technology assessment at hospitals: None of the hospitals conduct specific health technology assessment. They have a product review committee to evaluate new products they want to acquire. However, these are not formal committees, except in the public hospital, where it is still in an early stage of development. In the public hospital, this committee is part of the hospital structure and has a formal meeting scheduled for once a month.

Two hospitals, Private $\mathrm{C}$ and Public, use standardized product evaluation forms when they need to assess a new product prior to introduction. On the other hand, Private A and Private B have a range of different forms depending on the purpose of the evaluation, for example, a 'new or alternative product trial' form and 'consumable product evaluation' form.

However, all hospitals in the study are using business case analyses to analyse the cost effectiveness of the more expensive products. Table 2 summarises the criteria the hospitals use for analysis and compares these with the mini-HTA criteria. 
Am. Med. J. 2 (2): 72-78, 2011

Table 1: Hospital demographics

\begin{tabular}{lllll}
\hline & Private A & Private B & Private C & Public \\
\hline Bed No. & 286 beds & 149 beds & 58 beds & 302 beds \\
Staff No. & 1450 employees & 485 employees & 110 employees & 1972 employees \\
Services & Medical and & Medical and & Medical and & Medical and \\
& Surgical Services & Surgical Services & Surgical Services & Surgical Services \\
\hline
\end{tabular}

Table 2: Summary of criteria used for health technology assessment

\begin{tabular}{|c|c|c|c|c|c|}
\hline & Mini-HTA & Private A & Private $\mathrm{B}$ & Private $\mathrm{C}$ & Public \\
\hline $\begin{array}{l}\text { Standardize form } \\
\text { for HTA checklist }\end{array}$ & Yes & No & No & Yes & Yes \\
\hline $\begin{array}{l}\text { Main issues assess } \\
\text { in the form: }\end{array}$ & $\begin{array}{l}\text { Technology } \\
\text { Patient } \\
\text { Organization } \\
\text { Economy }\end{array}$ & $\begin{array}{l}\text { Technology } \\
\text { Workplace health } \\
\text { and safety issues } \\
\text { Change } \\
\text { management } \\
\text { Cost analysis }\end{array}$ & $\begin{array}{l}\text { Technology } \\
\text { Workplace health } \\
\text { and safety issues } \\
\text { Cost benefits } \\
\text { Clinical and } \\
\text { financial risk }\end{array}$ & $\begin{array}{l}\text { Technology } \\
\text { Projected use } \\
\text { of the product } \\
\text { Quality and safety } \\
\text { Cost analysis }\end{array}$ & $\begin{array}{l}\text { Safety } \\
\text { Effectiveness } \\
\text { Clinical Feasibility } \\
\text { Issues related to access } \\
\text { and equity and legal } \\
\text { and ethical implication } \\
\text { Cost analysis }\end{array}$ \\
\hline
\end{tabular}

Decision making process: The respondents from Private A and Private B described the current process as "informal", though they do have a committee to review the requests. Respondents from Private $\mathrm{C}$ and Public hospital described the processes in their hospitals as "formal".

The costs of the new technologies determine the complexity of the decision processes. The department managers have the authority to make decisions for new technologies that cost less than AUD $\$ 1,000$. If the product costs are more than AUD $\$ 1,000$, the requester has to prepare a business case and present it to the executive committee. There are also product review forms to be completed when requesting new technologies. If they are small cost items (less than AUD\$1,000) or simple modifications in procedure, usually they will just introduce the new technologies immediately at the department level without further discussion with senior executives. However, for expensive new technologies or technologies or procedures that can affect business strategies, decision making is in the hands of senior executives. For both types of hospitals, the decisions were made collectively, but the final decisions are made by the General Managers in the private hospitals or Executive Director in the public hospital. In the public hospital, the ethics and legality of the technologies also affect the decisions.

For not-for-profit private hospitals, doctors have a major influence on decisions to introduce new technologies. The significance of the doctors' demands reflect the fact that the doctors bring customers to the hospitals. The patients or customers will usually go where their doctors go. As one interviewee put it:

"a fair amount of the new technology is introduced to us from the doctors and they can sometime put pressure on us to buy the new technology."

As for public hospitals, doctors are also the main instigators but their requests can be declined if the technology that the doctor requests is too expensive, or is not yet clinically proven. Other instigators in public hospitals include the need to replace out-dated technologies; and also instructions from the State Health Department (Queensland Health) that the hospital has to follow.

The processes can flow from top - down or from the bottom - up. Both types of hospitals are part of a bigger parent organization. Sometimes, the parent organizations at corporate level will instruct these hospitals to acquire certain new technology and they will have to follow such a top-down decision. However, the requests can come from doctors or nurses. The requesters typically go to the department head and make their request. Usually the department head will ask the requester to fill up the specific form, such as product review form, and find out all the information needed, such as the cost-benefit, the product quality, the effectiveness, and the safety issues. Then the form will go back to the department head, and if the product request is an inexpensive product, is not complicated to be introduced and is low risk, the department head will make a decision at his/her level.

However, if the product involved is high risk and is complicated it will go through the product review committee in the public hospital and executive committee in the private hospitals. Furthermore, if the cost involved is high, the requester must prepare a business case, which usually goes further up the organizational structure to the corporate level of the parent organizations. In private hospitals, the executive committee has the power to make decisions even 
though the cost involved is high, but for the public hospital, the decision is made at the corporate level of the parent organization, if it involves high cost items.

The evaluation mechanism: the private hospitals stated 'cost and business strategy' as their most important criterion for evaluation, in contrast to the public hospital that stated 'patients' safety' as the most important criterion. All hospitals stated the requestor(s) and the vendor(s) as their sources of information, nevertheless other sources of information were also quoted, such as other hospitals, journal articles, conferences and seminars:

"We contact other hospitals.... We do that frequently... and ask them about the equipment that we have nothing to do with before... we go through the references, read articles...."

Neither private nor public hospitals have a specific or formal guideline for new health technology evaluation. In the public hospital, the guidelines are largely undeveloped and they are still at the design stage of the evaluation form.

Perceptions of Health Technology Assessment (HTA): At the public hospital, HTA is a requirement for new health technology decision making. Queensland Health has produced a policy stating that decision making for health technology must follow HTA guidelines, however it is still very new and the implementation stage is still in its infancy. In private practice, the HTA is not a requirement for new health technology decision making, though participants agreed that HTA could be a valuable tool for decision making.

Decision makers from both types of hospitals were unfamiliar with the HTA and mini-HTA; both the term and the guidelines. However, the decision makers have searched for good guidelines or forms that they can adapt in order to have a more structured decision making process. They believe it would be a good idea to make HTA a formal requirement in the decisionmaking process for introducing new health technologies in their hospitals. They also state that HTA has met their expectation of what HTA should be:

"Yes, we pretty much get what we want about new technologies from the health technology assessment articles and research reports".

Facilitators and barriers for new health technology adoption and future improvement: The majority of the respondents from both types of hospitals stated that an open and innovative organisational culture was critical as a facilitator for the adoption of new health technologies, whereas limited resources and space were seen as major barriers. Respondents did not view human resources as a factor, because staff can be trained and up-skilled:

"The culture of the organisation is very open and I think that the staff members and the people who...like doctors and everything ... they're also quite comfortable in suggesting or being willing to try, they don't feel hampered in any way that they will get a 'no' answer. They know they will have a collaborative and sort of open discussion about new technology. So the culture is one of ... I guess... supportive of new equipment and procedures when those things come in".

However, the participants from the Public hospital believed that bureaucracy is also an important barrier to the introduction of new technologies. Resistance to change among the staff is another barrier.

In terms of future improvement, $90 \%$ of the decision makers in the Private hospitals believe that the decision making process should be more structured, because structured processes ensure that the decisions are supported by facts and will reduce unfairness and prejudiced responses.

Participants also spoke about timely information, they want the information be there when they need it, not one of two years afterwards because the technologies are rapidly change and after one or two years there will undoubtedly be a newer technology available.

Participants also believe it would be valuable if they could get information on new technology from an independent body, such as HTA agencies.

The participants from public hospitals suggested that the product review committee members in their hospital should have more variation in membership so as to include representatives from doctors, nurses, pharmacies, and administrators, and not just from nurses.

\section{DISCUSSION}

The public hospital in this study has taken steps to ensure more evidenced-based decision making. Based on instructions from Queensland Health, the executives at the public hospital took the initiative to develop an HTA guide or form to assist in new health technology decisions and priority settings. The evidence they sought was patient-oriented. 
In the not-for-profit private hospitals, however, the processes are 'informal' and many aspects of the decision making process did not comply with evidencebased decision making. For example, the evidence taken under consideration are not comprehensive, with cost and business strategies being the key deciding factors.

The impact of HTA as a support tool for decision makers at institutional level is still relatively minimal. Most decision makers, private and public, are not aware that there are independent bodies, such as HTA agencies worldwide where they can get unbiased information on health technologies. Such international HTA bodies as International Network of Agencies for Health Technology Assessment (INAHTA) and Health Technology Assessment International (HTAI) are unfamiliar to them. On the other hand, they are more familiar with agencies in Australia such as the Therapeutic Goods Administration (TGA), Medical Services Advisory Committee (MSAC) and Pharmaceutical Benefit Advisory Committee (PBAC), because these agencies are a regulatory agencies and usually they have to comply with these agencies regulations regarding health technologies before they can introduce them in their hospitals. The limited use of such evidence might lead to less than well informed decision making, which, in turn, might have an impact on patients.

More effective strategies for disseminating the HTA evidence to decision makers should be employed by HTA agencies and associated regulatory bodies. More structured decision making processes for introducing new health technologies at the hospital level should be developed and introduced. Hospital authorities can use a formal HTA form, guide or checklist to assess new technologies but such a form should reflect the reasoning behind HTA, namely: technology, patient, organization and economy. Hospitals could refer to the 'mini-HTA' form as a bestpractice guideline when creating their own HTA form.

A recent initiative from Queensland Health instructs the hospitals under its jurisdiction to create a standardised procedure for decision making processes to introduce new health technologies. As yet, however, no standardised process has been introduced and every hospital must create its own process, based on the available best practice. In Danish hospitals, it has been proven that mini-HTA is a good tool to address this issue (Kidholm et al., 2009). It can be argued that the mini-HTA form should be introduced more rigorously, because it is adaptable and can become the best available tool for decision making processes to introduce new health technologies.
Hospitals also should have at least an HTA officer if not one specific unit or department. This officer should have a good knowledge of HTA, such as what it is, how to conduct HTA at the hospital and where to find the required information. This position could provide an independent assessment for new technologies that the hospital may want to introduce. With this in mind, the information gathered will not be biased and the more evidence based decision processes will be employed.

\section{CONCLUSION}

There is still a long way to go to develop an efficient and effective process to translate research evidence into practice. There are, however, a number of strategies that the regulatory bodies and HTA agencies can use to make sure the new health technologies are introduced in the best interests of the patients. One such strategy is to introduce mini-HTA or hospital based HTA. This would mean that the assessment hospitals undertake on new health technology before deciding to introduce it, would be based on the same criteria as the HTA reasoning: technology, patient, organisation, and economics, and not just for economic or profit oriented reasons alone.

Evidence from Demark and other countries suggests a demonstrable need for local HTA tools at hospitals (Ehlers et al., 2006). The mini-HTA can become such a tool with a format that is flexible enough for local adaptation, to meet local needs (Vestergaard $e t$ al., 2005). Health authorities and parent or corporate bodies overseeing the private health sector should strongly promote the introduction of mini-HTA in hospitals, public as well as private.

\section{ACKNOWLEDGMENT}

Part of the research reported in this article has been published elsewhere. The first author is currently a $\mathrm{PhD}$ candidate at School of Public Health, Griffith University, Queensland. She would like to thank her supervisors, her funders (University Technology MARA, Malaysia and Ministry of Higher Education, Malaysia), and also the participating hospitals and all participants.

\section{REFERENCES}

Australian Government, 2009. Review of Health Technology Assessment in Australia: A Discussion Paper. Department of Health and Ageing. 
Banta, D. and W. Oortwijn, 2000. Conclusion: Health technology assessment and health care in the European Union. Int. J. Technol. Assess. Health Care, 16: 626-635.

Ehlers, L., M. Vestergaard, K. Kidholm, B. Bonnevie and P.H. Pedersen et al., 2006. Doing mini-health technology assessments in hospitals: A new concept of decision support in health care. Int. J. Technol. Assess. Health Care, 22: 295-301. DOI: 10.1017/S0266462306051178

Gagnon, M.P., E. Sanchez and J.M.V. Pons, 2006. Integration of health technology assessment recommendations into organizational and clinical practice: A case study in Catalonia. Int. J. Technol. Assess. Health Care, 22: 169-176. DOI: 10.1017/S0266462306050987

Gallego, G., 2006. Access to High Cost Medicines in Australian Hospitals. Faculty of Pharmacy, The University of Sydney, Sydney.

Gallego, G., S. Fowler and K.V. Gool, 2008. Decision makers' perceptions of health technology decision making and priority setting at the institutional level. Aust. Health Rev., 32: 520-527. DOI: 10.1071/AH080520

Haas, M., J. Hall, R. Viney, G. Gallego and S. Goodall et al., 2008. A Model for Best Practice HTA. Centre for Health Economics Research and Evaluation, Faculty of Business, University of Technology, Sydney.

Haines, A., S. Kuruvilla and M. Borchert, 2004. Bridging the implementation gap between knowledge and action for health. Bull. World Health Organ., 82: 724-731.

HBSP, 2006. Harvard Business Essentials, Decision Making: 5 Steps to Better Results. 1st Edn., Harvard Business Press, ISBN-10: 1591397618, pp: 168 .

Hivon, M., P. Lehoux, J.L. Denis and S. Tailliez, 2005. Use of health technology assessment in decision making: Coresponsibility of users and producers? Int. J. Technol. Assess. Health Care, 21: 268-275. PMID: 15921069

Johnson-Masotti, A.P. and K. Eva, 2005. A DecisionMaking Framework for the Prioritization of Health Technologies. Queen's University, Kingston, Canada.

Kapiriri, L. and O.F. Norheim, 2004. Criteria for priority-setting in health care in Uganda: exploration of stakeholders' values. Bull. World Health Organ., 82: 172-179. PMID: 15112005
Kidholm, K., L. Ehlers, L. Korsbek, R. Kjaerby and M. Beck, 2009. Assessment of the quality of miniHTA. Int. J. Technol. Assess. Health Care, 25: 4248. DOI: $10.1017 /$ S0266462309090060

Leggat, S.G., W. Scheil, H. Williams and K. Kerin, 2006. Tools for priority setting: Lessons from South Australia. Aust. Health Rev., 30: 65-72. PMID: 16448379

Lehoux, P., J.L. Denis, S. Tailliez and M. Hivon, 2005. Dissemination of health technology assessments: Identifying the visions guiding an evolving policy innovation in Canada. J. Health Polit. Policy Law, 30: 603-642. DOI: 10.1215/03616878-30-4-603

McGregor, M., 2006. What decision-makers want and what they have been getting. Value Health, 9: 181185. DOI: 10.1111/j.1524-4733.2006.00098.x

Mullen, P.M., 2004. Quantifying priorities in healthcare: Transparency or illusion? Health Serv. Manage. Res., 17: 47-48. DOI: 10.1258/095148404322772723

Noorani, H.Z., D.R. Husereau, R. Boudreau and B. Skidmore, 2007. Priority setting for health technology assessments: A systematic review of current practical approaches. Int. J. Technol. Assess. Health Care, 23: 310-315. DOI: 10.1017/S026646230707050X

Perrott, B. and R. Hughes, 2005. Marketing dynamics in the Australian private hospital industry. The ANZMAC 2005 Conference: Business Interaction, Relationships and Networks.

Sanders, J.M., 2002. Challenges, choices and Canada. Int. J. Technol. Assess. Health Care, 18: 199-202. DOI: 10.1017/S0266462302000211

Sorenson, C., M. Drummond, F.B. Kristensen and R. Busse, 2008. How can the impact of health technology assessments be enhanced? Proceedings of the WHO European Ministerial Conference on Health Systems, Jun. 25-27, Tallinn, Estonia, pp: $1-23$.

Stevens, A., R. Milne and A. Burls, 2003. Health technology assessment: History and demand. J. Public Health Med., 25: 98-101. DOI: 10.1093/pubmed/fdg022

Vestergaard, M., L. Ehlers, K. Kidholm, P.H. Pedersen and B. Bonnevie et al., 2005. DK: Introduction to mini-Health Technology Assessment (HTA). The National Board of Health, Danish Centre for Evaluation and Health Technology Assessment, Copenhagen. 